\title{
Cancer-associated fibroblasts, matrix metalloproteinase-9 and lymphatic vessel density are associated with progression from adenocarcinoma in situ to invasive adenocarcinoma of the lung
}

\author{
CHENG CHEN $^{1 *}$, WU-JUN LI ${ }^{1 *}$, JING-JING WENG ${ }^{1}$, ZHI-JUN CHEN $^{1}$, YUAN-YUAN WEN $^{2}$, \\ TAO DENG ${ }^{2}$, HAN-BO LE ${ }^{1}$, YONG-KUI ZHANG ${ }^{1}$ and BIN-JIE ZHANG ${ }^{1}$ \\ Departments of ${ }^{1}$ Cardio-Thoracic Surgery and ${ }^{2}$ Pathology, Zhoushan Hospital, Zhoushan, Zhejiang 316000, P.R. China
}

Received February 24, 2020; Accepted July 16, 2020

DOI: 10.3892/ol.2020.11991

\begin{abstract}
The present study aimed to investigate the roles of cancer-associated fibroblasts (CAFs), matrix metalloproteinase-9 (MMP-9) and lymphatic vessel density (LVD) during the progression from adenocarcinoma in situ (AIS) to invasive lung adenocarcinoma (IAC). A total of 77 patients with stage 0 -IA lung adenocarcinoma were enrolled. The expression levels of $\alpha$-smooth muscle actin, MMP-9 and D2-40 were immunohistochemically analyzed. Survival analysis was performed using the Kaplan-Meier method. In the non-invasive component, the proportion of CAFs and the expression levels of MMP-9 increased from AIS to IAC; however, the LVD was not significantly different. CAFs were positively correlated with levels of MMP-9. The LVD had no significant correlation with CAFs and MMP-9. In the invasive component, CAFs, MMP-9 and LVD were significantly higher in IAC compared with in minimally invasive adenocarcinoma. CAFs, MMP-9 and LVD were all positively correlated with each other. The micropapillary subtype in IAC was associated with overall survival (OS). The LVD in IAC, but not MMP-9 and CAFs, was associated with OS. CAFs, MMP-9 and LVD were involved in the progression from AIS to IAC. CAFs exhibited a strong association with MMP-9 levels in the non-invasive and invasive components. The increase in the proportion of CAFs and the expression levels of MMP-9 may have been an early event before the adenocarcinoma became invasive. Once the adenocarcinoma was invasive, the LVD served an important role in tumor invasion and metastasis, and hence may be used as a prognostic marker of poor OS in stage IA IAC.
\end{abstract}

Correspondence to: Dr Bin-Jie Zhang, Department of Cardio-Thoracic Surgery, Zhoushan Hospital, 739 Dingshen Road, Zhoushan, Zhejiang 316000, P.R. China

E-mail: zbj668508@163.com

*Contributed equally

Key words: adenocarcinoma in situ, cancer-associated fibroblasts, lymphatic vessel density, matrix metalloproteinase-9, minimally invasive adenocarcinoma

\section{Introduction}

Lung cancer is the most common cause of cancer-associated mortality worldwide (1). In 2012, there were 1.82 million new lung cancer cases (accounting for $12.9 \%$ of the total newly diagnosed cancers) and 1.59 million lung cancer deaths (accounting for $19.4 \%$ of the total cancer deaths) globally (1). Non-small cell lung cancer (NSCLC) accounts for $\sim 85 \%$ of lung cancer cases $(1,2)$. Lung adenocarcinoma represents the most common histological subtype of NSCLC (2). The number of diagnosed small-sized lung adenocarcinomas has now increased due to the improvement of imaging techniques (3). However, the clinical outcome remains unsatisfactory even after curative surgery.

In 2011, a novel lung adenocarcinoma classification was advocated by the International Association for the Study of Lung Cancer, the American Thoracic Society and the European Respiratory Society. Adenocarcinoma in situ (AIS) is defined as a $\leq 3 \mathrm{~cm}$ tumor with a pure lepidic pattern without invasion; minimally invasive adenocarcinoma (MIA) is defined as a $\leq 3 \mathrm{~cm}$ tumor with a lepidic-predominant pattern with $\leq 0.5 \mathrm{~cm}$ invasion; and invasive adenocarcinoma (IAC) is further classified according to the predominant subtype: Lepidic, papillary, acinar, solid or micropapillary-predominant adenocarcinoma (4). The progression of lung adenocarcinoma is thought to develop in a stepwise manner (5), but the underlying mechanism is not well elucidated.

Patients with AIS/MIA have an $\sim 100 \%$ disease-free survival rate when the tumors are radically resected (4). However, a considerable proportion of patients with stage I IAC ultimately exhibit recurrence (6). Therefore, improving the knowledge of the mechanisms underlying the development and progression of lung adenocarcinoma is essential, which may lead to novel risk classifications for lung adenocarcinoma and to the development of more effective treatment strategies.

The tumor tissue is composed of cancer cells, as well as various types of stromal cells, including endothelial cells, fibroblasts and immune cells, in addition to the extracellular matrix (ECM) they produce, blood vessels and lymphatics, which together create a specific tumor microenvironment critical for tumor progression $(7,8)$. Cancer-associated fibroblasts (CAFs) are representative cells of the tumor microenvironment (9). 
Extensive clinical and experimental evidence has suggested that CAFs may promote tumor progression in various ways, including immune response regulation (10), angiogenesis (11) and ECM remodeling (12). Studies have demonstrated that CAFs are associated with a poor prognosis in lung adenocarcinoma $(13,14)$.

An important characteristic of the aggressiveness of tumor cells is their ability to remodel and degrade the ECM using specific enzymes (15). Matrix metalloproteinase-9 (MMP-9) is a member of the zinc-containing endopeptidase family and the most important component in tumor tissue remodeling (16). MMP-9 can degrade types IV, V, VII, IX and X collagen, elastin, fibrin, fibrinogen and plasminogen, thus enabling tumor cell invasion and metastasis (16). Furthermore, MMP-9 can influence the vascular endothelial growth factor (VEGF)-mediated development of an angiogenic vasculature $(17,18)$. High MMP-9 expression in NSCLC has an independent prognostic value for the diagnosis of distant metastasis or local recurrence (19).

In addition to the invasive ability of tumor cells, lymphangiogenesis, referring to the growth of lymphatic vessels, is considered to be the initial step and basic requirement for lymphatic metastasis (20). Lymphatic vessel density (LVD) is the parameter most frequently used to quantify tumor lymphangiogenesis (20). Studies have demonstrated that LVD is an independent prognostic factor in $\operatorname{NSCLC}(21,22)$.

However, previous studies have focused mainly on the advanced stages of NSCLC, and only a few AIS and MIA cases were examined in these studies $(13,14,19,21,22)$. Therefore, the role of CAFs, MMP-9 and LVD, and their association in the progression from AIS to IAC remains mostly unknown. In the present study, immunohistochemical (IHC) analysis was used to evaluate the importance of CAFs, MMP-9 and LVD in both non-invasive (lepidic) and invasive (other) tumor components in the progression from AIS to IAC. Additionally, the potential correlations among these parameters and their prognostic values were investigated.

\section{Materials and methods}

Patients. A total of 77 patients with stage 0-IA lung adenocarcinoma who underwent complete resection at the Department of Thoracic Surgery of Zhoushan Hospital (Zhoushan, China) between January 2013 and December 2013 were included in the present retrospective study. Mean age at the time of surgery was 62 years (age range: $34-83$ years) and the mean follow-up period was 57.3 months (range: 13-60 months). The clinicopathological characteristics of patients are summarized in Table I. The adenocarcinoma subtypes were classified according to the World Health Organization classification (fourth edition) (23). Each component (lepidic, papillary, acinar, solid and micropapillary) presented in MIA or $\geq 5 \%$ in IAC was recorded and assessed in individual cases. Breast cancer tissue from a 55-year-old female patient who underwent radical mastectomy at Zhoushan Hospital (Zhoushan, China) in 2013 was used as the positive control for the present study. The present study was approved by the Institutional Review Board of Zhoushan Hospital. The informed consent from patients was waived by the Institutional Review Board of Zhoushan Hospital due to the retrospective nature of the study.
IHC analysis. The resected tissue specimens were fixed in $10 \%$ formalin for $24 \mathrm{~h}$ embedded in paraffin, stored at room temperature and cut into $4-\mu \mathrm{m}$-thick serial sections. The sections were deparaffinized in xylene and rehydrated through graded alcohol (100\% alcohol, $5 \mathrm{~min}$; 95\% alcohol, $5 \mathrm{~min}$ and $75 \%$ alcohol, $5 \mathrm{~min}$ ) and deionized water, and then incubated with $3 \% \mathrm{H}_{2} \mathrm{O}_{2}$ at room temperature for $10 \mathrm{~min}$ to inhibit endogenous peroxidase activity. After extensive washing with PBS, the sections were further treated with $2 \%$ normal goat serum (OriGene Technologies, Inc.) in PBS for $30 \mathrm{~min}$ at room temperature to block non-specific binding. Next, the sections were rinsed with PBS three times and then incubated with primary antibodies against MMP-9 (1:300; cat. no. 13667S; Abcam), D2-40 (1:200; cat. no. ab77854; Abcam) and $\alpha$-smooth muscle actin ( $\alpha$-SMA; 1:600; cat. no. 19245S, Cell Signaling Technology, Inc.) overnight at $4^{\circ} \mathrm{C}$. The sections were then incubated with the HRP labeled polymer conjugated with secondary antibody (1:200; cat. no. PV-6000; Universal immunohistochemical kit, OriGene Technologies, Inc.) at room temperature for $20 \mathrm{~min}$. For color development, the sections were incubated with $2 \%$ DAB solution (DAB detection kit, OriGene Technologies, Inc.) for $5 \mathrm{~min}$ at room temperature. Each section was counterstained with hematoxylin for $2 \mathrm{~min}$ at room temperature, dehydrated by sequential immersions in $70 \%$ ethanol (1 min) and 100\% ethanol (twice for $1 \mathrm{~min}$ ) and finally mounted. Positive controls were breast carcinoma tissues, which are known to express MMP-9, D2-40 and $\alpha$-SMA. For negative controls, the primary antibody was replaced with PBS.

IHC staining was assessed independently by two pathologists with $>10$ years of experience using a light microscope, who were blinded to all clinicopathological features. When the evaluation results differed, a final consensus was reached by revaluation and discussion. The non-invasive and invasive components were determined based on hematoxylin and eosin staining according to the international multidisciplinary classification of lung adenocarcinoma (6). The staining score of each component was evaluated for every sample. The most densely populated staining areas (hot spot) were first identified under a magnification, x40 and then magnification, x400 was used to calculate staining scores. The mean value of three hot spots was considered the representative of one component. For comparing the non-invasive and invasive components, the highest score of one invasive component was considered the representative of the case. The area fraction of CAFs was defined as the ratio of the $\alpha$-SMA+ area to the total area of the microscopic field using ImageJ software version 1.48 (National Institutes of Health) $(24,25)$. The expression levels of MMP-9 were evaluated based on the staining intensity and the percentage of positive cells. The scores for the percentage of positive cells were as follows: $0,0-5 ; 1,6-25 ; 2,26-50$; $3,51-75$; and $4,76-100 \%$ (26). The staining intensity scoring was as follows: 0 , absent=no staining; 1 , weak=faint yellow; 2 , moderate=brown; and 3, strong=dark brown. The final staining scores were calculated by multiplying the scores for the percentage of positive cells and staining intensity, with the final scores ranging from 0 to 12 . D2-40 positive single endothelial cell or cell cluster, separated from adjacent lymphatic vessels, tumor cells and connective tissue elements, regardless of the presence of lumen was counted as one lymphatic vessel. 
Table I. Summary of clinicopathological characteristics.

\begin{tabular}{|c|c|c|c|c|c|}
\hline Variables & AIS (n=9) & $\operatorname{MIA}(n=24)$ & IAC $(n=44)$ & P-value ${ }^{a}$ & P-value ${ }^{b}$ \\
\hline Age, years & & & & $0.38^{c}$ & 0.67 \\
\hline$\leq 65$ & 8 & 16 & 27 & & \\
\hline$>65$ & 1 & 8 & 17 & & \\
\hline Sex & & & & $>0.999^{c}$ & 0.13 \\
\hline Male & 4 & 9 & 25 & & \\
\hline Female & 5 & 15 & 19 & & \\
\hline Smoking & & & & $>0.999^{c}$ & $0.06^{\mathrm{c}}$ \\
\hline No & 7 & 20 & 26 & & \\
\hline Yes & 2 & 4 & 18 & & \\
\hline \multicolumn{6}{|c|}{ Carcinoembryonic antigen, $\mathrm{ng} / \mathrm{ml}$} \\
\hline$\leq 5$ & 9 & 22 & 39 & $>0.999^{c}$ & $>0.999^{c}$ \\
\hline$>5$ & 0 & 2 & 5 & & \\
\hline Tumor size, $\mathrm{cm}$ & & & & $>0.999^{c}$ & $<0.01^{\mathrm{c}, \mathrm{d}}$ \\
\hline$\leq 2$ & 9 & 23 & 28 & & \\
\hline$>2$ and $\leq 3$ & 0 & 1 & 16 & & \\
\hline Location & & & & $0.03^{\mathrm{c}}$ & $0.37^{\mathrm{c}}$ \\
\hline Right upper lobe & 4 & 9 & 20 & & \\
\hline Right middle lobe & 0 & 2 & 3 & & \\
\hline Right lower lobe & 3 & 4 & 6 & & \\
\hline Left upper lobe & 0 & 9 & 10 & & \\
\hline Left lower lobe & 2 & 0 & 5 & & \\
\hline Vascular invasion & & & & - & $0.54^{\mathrm{c}}$ \\
\hline Absent & 9 & 24 & 42 & & \\
\hline Present & 0 & 0 & 2 & & \\
\hline
\end{tabular}

${ }^{\mathrm{a} A I S}$ vs. MIA; ${ }^{\mathrm{b}}$ MIA vs. IAC; ${ }^{\mathrm{c}}$ Fisher's exact test; ${ }^{\mathrm{d}} \mathrm{P}<0.025$ after Bonferroni correction. P-values were calculated by $\chi^{2}$ test unless indicated otherwise. AIS, adenocarcinoma in situ; MIA, minimally invasive adenocarcinoma; IAC, invasive adenocarcinoma.

Statistical analysis. Statistical analysis was performed using SPSS version 18.0 (SPSS, Inc.). The data are expressed as the mean \pm standard deviation. Categorical variables were compared using the $\chi^{2}$ test or Fisher's exact test, as appropriate. Continuous variables were compared using the independent-samples Student's t-test (for differences between 2 groups) or a one-way ANOVA followed by Fisher's least significant difference (LSD) post hoc test (for comparisons among $>2$ groups). Variables in different components of one tumor were compared using the paired-samples Student's t-test. The correlation between continuous variables was analyzed using Pearson's correlation coefficient. Overall survival (OS) was defined as the time between surgery and mortality or last observation. OS was analyzed using the Kaplan-Meier method and the log-rank test. $\mathrm{P}<0.05$ (two-sided) was considered to indicate a statistically significant difference. Multiple comparisons were made using the Bonferroni correction as appropriate $(\mathrm{P}<0.05 / \mathrm{n})$.

\section{Results}

Clinicopathological characteristics of patients with AIS, $M I A$ and IAC. The clinicopathological characteristics for all 77 patients are summarized in Table I. No significant differences in age, sex, smoking, carcinoembryonic antigen level, tumor location and vascular invasion were observed among the three groups. However, patients with IAC had a significantly larger tumor size compared with patients with MIA $(\mathrm{P}<0.01)$.

Differences in CAFs, MMP-9 and LVD in the non-invasive and invasive components of tumors of patients with AIS, $M I A$ and IAC. The representative samples of IHC staining are shown in Fig. 1. Within the non-invasive component, the mean area percentage for CAFs was $6.04 \pm 3.00,9.70 \pm 3.28$ and $12.10 \pm 4.44$ in patients with AIS, MIA and IAC, respectively. The mean staining score for MMP-9 was $1.56 \pm 1.74$, $4.00 \pm 2.09$ and $5.23 \pm 1.98$ in patients with AIS, MIA and IAC, respectively. The mean LVD count was $4.33 \pm 1.66,3.33 \pm 1.58$ and 3.95 \pm 1.68 in patients with AIS, MIA and IAC, respectively (Table II). One-way ANOVA followed by the LSD test revealed that the proportion of CAFs and the expression levels of MMP-9 significantly increased from AIS to IAC (CAFs, $\mathrm{F}=8.497$ and $\mathrm{P}=0.001$; AIS vs. MIA, $\mathrm{P}=0.016$; AIS vs. IAC, $\mathrm{P}<0.001$; MIA vs. IAC, $\mathrm{P}=0.035$; MMP-9, $\mathrm{F}=10.908$ and $\mathrm{P}<0.001$; AIS vs. MIA, $\mathrm{P}=0.003$; AIS vs. IAC, $\mathrm{P}<0.001$; 


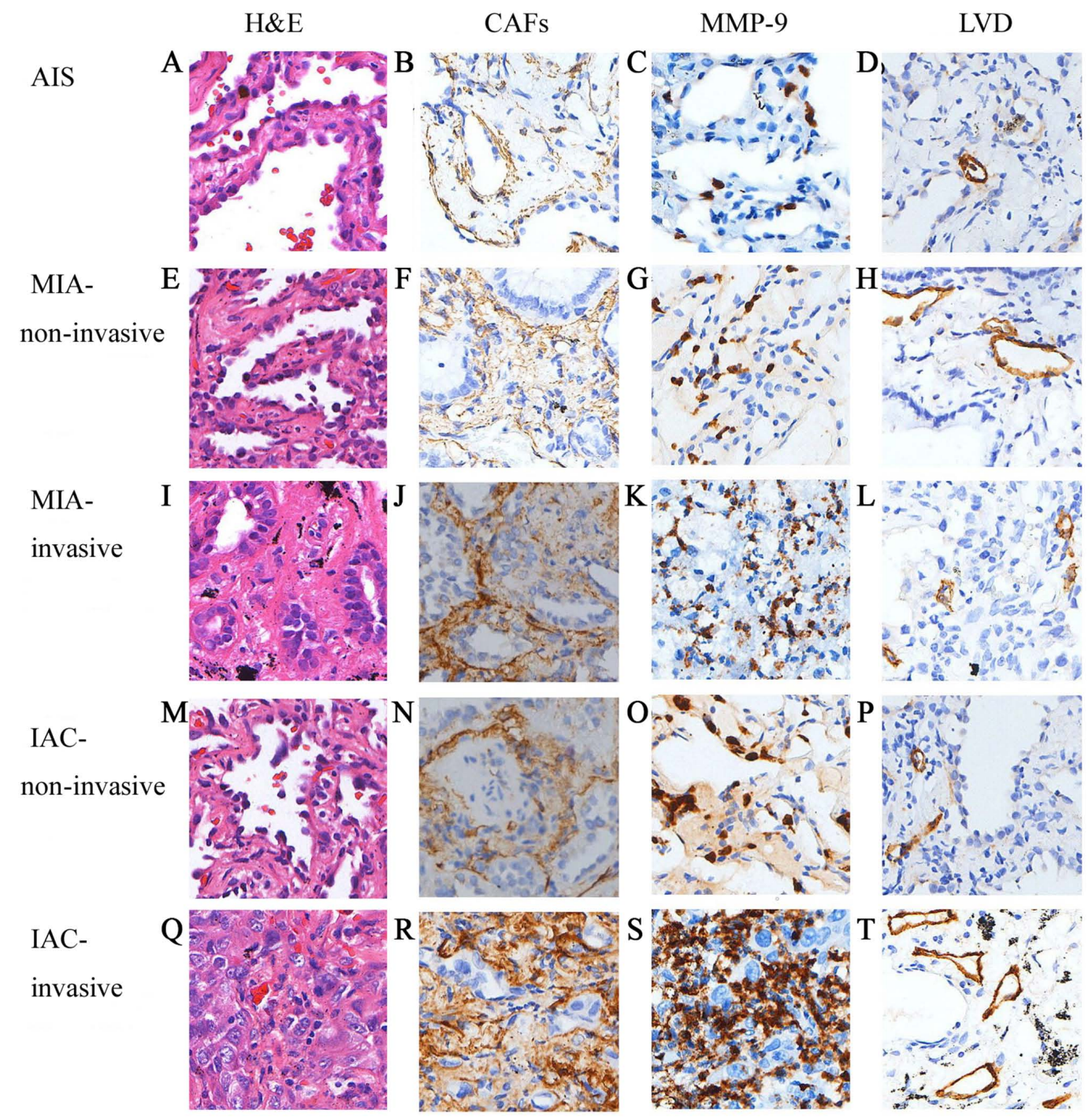

Figure 1. Representative immunostaining results for H\&E, CAFs, MMP-9 and LVD. Staining results in (A-D) AIS, (E-H) non-invasive component of MIA, (I-L) invasive component of MIA, (M-P) non-invasive component of IAC and (Q-T) invasive component of IAC. Magnification, x200. H\&E, hematoxylin and eosin; CAFs, cancer-associated fibroblasts; MMP-9, matrix metalloproteinase-9; LVD, lymphatic vessel density; AIS, adenocarcinoma in situ; MIA, minimally invasive adenocarcinoma; IAC, invasive adenocarcinoma.

MIA vs. IAC, $\mathrm{P}=0.042$; Tables II and III). The LVD was not significantly different while progressing from AIS to IAC $(\mathrm{F}=1.532$ and $\mathrm{P}=0.226$; AIS vs. MIA, $\mathrm{P}=0.123$; AIS vs. IAC, $\mathrm{P}=0.56$; MIA vs. IAC, $\mathrm{P}=0.203$; Tables II and III). Within the invasive component, the mean area percentage for CAFs was $11.04 \pm 4.33$ and $13.43 \pm 4.15$ in MIA and IAC, respectively. The mean staining score for MMP-9 was 4.58 2.26 and $5.68 \pm 1.86$ in MIA and IAC, respectively. The mean LVD was $4.83 \pm 2.41$ and 5.45 \pm 2.87 in MIA and IAC, respectively. CAFs $(\mathrm{P}=0.001)$, MMP-9 $(\mathrm{P}=0.004)$ and LVD $(\mathrm{P}=0.013)$ all significantly increased from MIA to IAC (Table II).

Differences in CAFs, MMP-9 and LVD between the non-invasive and invasive components of tumors of patients with MIA and IAC. Of the 44 IAC cases, 22 also had a non-invasive component (lepidic) and thus were included in this comparison. The scores of CAFs, MMP-9 and LVD were all significantly higher in the invasive component compared with in the non-invasive component in both MIA and IAC $(\mathrm{P}<0.05$; Table IV).
Differences in CAFs, MMP9 and LVD in the invasive subtypes of IAC samples. The scores of CAFs, MMP-9 and LVD in the subtypes of IAC in 44 cases (since most cases of lung adenocarcinoma exhibited mixed subtypes, 32 papillary, 23 acinar, 11 solid and 10 micropapillary) were compared to further explore the differences in CAFs, MMP-9 and LVD in the invasive subtypes of IAC samples. The cases with solid and micropapillary patterns were fewer, and hence included in the solid + micropapillary group, while the other two patterns were included in the acinar + papillary group. As shown in Table V, the scores of CAFs, MMP-9 and LVD were all significantly higher in the solid + micropapillary group compared with in the acinar + papillary group $(\mathrm{P}=0.011, \mathrm{P}=0.045$ and $\mathrm{P}<0.001$, respectively).

Correlations between CAFs, MMP-9 and LVD in the non-invasive and invasive components. Considering all of the samples as a whole, CAFs were positively correlated with MMP-9 in the non-invasive component $(\mathrm{r}=0.398 ; \mathrm{P}=0.003$; Fig. 2A). No statistically significant correlation was observed 
Table II. Differences in CAFs, MMP-9 and LVD in the non-invasive and invasive components of patients with AIS (n=9), $\operatorname{MIA}(n=24)$ and IAC $(n=22)$.

\begin{tabular}{|c|c|c|c|c|c|c|c|c|}
\hline \multirow[b]{2}{*}{ Variable } & \multicolumn{5}{|c|}{ Non-invasive component } & \multicolumn{3}{|c|}{ Invasive component } \\
\hline & AIS & MIA & IAC & F ratio & P-value & MIA & IAC & P-value ${ }^{b}$ \\
\hline CAFs & $6.04 \pm 3.00$ & $9.70 \pm 3.28$ & $12.10 \pm 4.44$ & 8.497 & 0.001 & $11.04 \pm 4.33$ & $13.43 \pm 4.15$ & 0.001 \\
\hline MMP-9 & $1.56 \pm 1.74$ & $4.00 \pm 2.09$ & $5.23 \pm 1.98$ & 10.908 & $<0.001$ & $4.58 \pm 2.26$ & $5.68 \pm 1.86$ & 0.004 \\
\hline LVD & $4.33 \pm 1.66$ & $3.33 \pm 1.58$ & $3.95 \pm 1.68$ & 1.532 & 0.226 & $4.83 \pm 2.41$ & $5.45 \pm 2.87$ & 0.013 \\
\hline
\end{tabular}

aOne-way ANOVA. bindependent-samples Student's t-test. CAFs, cancer-associated fibroblasts; MMP-9, matrix metalloproteinase-9; LVD, lymphatic vessel density; AIS, adenocarcinoma in situ; MIA, minimally invasive adenocarcinoma; IAC, invasive adenocarcinoma.

Table III. LSD post hoc test for CAFs, MMP-9 and LVD between groups of non-invasive components in patients with AIS, MIA and IAC.

\begin{tabular}{lccc}
\hline & AIS & MIA & IAC \\
\hline CAFs & & & \\
AIS & - & $\mathrm{P}=0.016$ & $\mathrm{P}<0.001$ \\
MIA & - & - & $\mathrm{P}=0.035$ \\
IAC & - & - & - \\
& AIS & MIA & IAC \\
MMP-9 & & & \\
AIS & - & $\mathrm{P}=0.003$ & $\mathrm{P}<0.001$ \\
MIA & - & - & $\mathrm{P}=0.042$ \\
IAC & - & - & - \\
& AIS & $\mathrm{MIA}$ & $\mathrm{IAC}$ \\
LVD & & & $\mathrm{P}=0.56$ \\
AIS & - & $\mathrm{P}=0.123$ & $\mathrm{P}=0.203$ \\
MIA & - & - & \\
IAC & - & - & \\
\hline
\end{tabular}

CAFs, cancer-associated fibroblasts; MMP-9, matrix metalloproteinase-9; LVD, lymphatic vessel density; AIS, adenocarcinoma in situ; MIA, minimally invasive adenocarcinoma; IAC, invasive adenocarcinoma.

between CAFs and LVD ( $\mathrm{r}=0.183 ; \mathrm{P}=0.181$; Fig. 2B), and between MMP-9 and LVD ( $r=0.238$; $\mathrm{P}=0.08$; Fig. 2C). In the invasive component, CAFs were positively correlated with MMP-9 (r=0.395; P=0.001; Fig. 2D) and LVD ( $\mathrm{r}=0.408$; $\mathrm{P}=0.001$; Fig. 2E). Additionally, MMP-9 was positively correlated with LVD (r=0.427; $\mathrm{P}<0.001$; Fig. 2F).

Associations of invasive subtypes, CAFs, MMP-9 and LVD with $O S$ in patients with IAC. All patients with AIS and MIA achieved a $100 \%$ disease-free survival rate in the present study (data not shown). Therefore, the prognostic value of clinicopathological variables, invasive subtypes, CAFs, MMP-9 and LVD in the 44 patients with IAC was investigated. The highest scores for CAFs, MMP-9 and LVD in the invasive component were used as the respective scores for the survival analysis. Patients were classified into high or low groups based on the median scores of CAFs, MMP-9 and LVD (6, 13.5 and 5 , respectively), and positive or negative invasive subtypes, an invasive subtype with a proportion $\geq 5 \%$ was defined as positive. A significant association was observed between patients with OS and LVD, patients with high LVD had a poorer OS rate compared with those with low LVD ( $\mathrm{P}=0.005$; Fig. 3E), but not with MMP-9 and CAFs $(\mathrm{P}=0.530$ and $\mathrm{P}=0.468$, respectively; Fig. 3F and G). The micropapillary pattern was associated with poor $\mathrm{OS}(\mathrm{P}=0.031$; Fig. $3 \mathrm{~A})$, while other patterns had no significant association with $\mathrm{OS}$ (solid, $\mathrm{P}=0.336$; papillary, $\mathrm{P}=0.090$; acinar, $\mathrm{P}=0.533$; Fig. 3B-D).

\section{Discussion}

The contribution of CAFs to the development of lung adenocarcinoma has been supported by clinical evidence and experimental models $(13,27,28)$. However, these previous studies have rarely examined non-invasive lesions, which may represent the initial stage of lung adenocarcinoma. Little is known about how CAFs differ in non-invasive and invasive lesions. Kawase et al (14) reported that CAFs were only detected in invasive adenocarcinoma and none were detected in non-invasive adenocarcinoma. The present study revealed that there were fewer CAFs in the non-invasive component than in the invasive component. Additionally, CAFs displayed a significant increase in both non-invasive and invasive components during the progression from AIS to IAC. It was hypothesized that CAFs may provide a more tumor-promoting microenvironment while progressing from AIS to IAC. However, Matsubara et al (29) revealed completely opposite results: Myofibroblasts positive for $\alpha$-SMA, which were named subepithelial myofibroblasts, were present in the non-invasive components, but scanty in the invasive components. The retention of subepithelial myofibroblasts in invasive lung adenocarcinoma was associated with an excellent prognosis in patients (29). Therefore, the specific phenotype, morphology and location of CAFs require further exploration.

Kodate et al (30) revealed that $1 / 11$ cases of bronchioloalveolar carcinoma expressed MMP-9 in the non-invasive component. Kanomata et al (31) investigated the mRNA expression levels of MMP-9 in different areas of mixed-type lung adenocarcinoma using in situ hybridization; the mRNA expression levels and the activity of MMP-9 were significantly higher in both non-invasive and invasive carcinomas compared with in tumor-free lung tissues. Consistent with 
Table IV. Differences in CAFs, MMP-9 and LVD between the non-invasive and invasive components in patient with MIA ( $\mathrm{n}=24)$ and $\operatorname{IAC}(\mathrm{n}=22)$.

\begin{tabular}{lcrrrrrr}
\hline & \multicolumn{3}{c}{ MIA } & & \multicolumn{3}{c}{ IAC } \\
\cline { 2 - 3 } Variable & Non-invasive & Invasive & P-value & & Non-invasive & Invasive & P-value \\
\hline CAFs & $9.70 \pm 3.28$ & $11.04 \pm 4.33$ & 0.019 & & $12.10 \pm 4.44$ & $13.43 \pm 4.15$ & 0.017 \\
MMP-9 & $4.00 \pm 2.09$ & $4.58 \pm 2.26$ & 0.013 & & $5.23 \pm 1.98$ & $5.68 \pm 1.86$ & 0.021 \\
LVD & $3.33 \pm 1.58$ & $4.83 \pm 2.41$ & $<0.001$ & & $3.95 \pm 1.68$ & $5.45 \pm 2.87$ & 0.027 \\
\hline
\end{tabular}

P-values calculated using a paired-samples Student's t-test. CAFs, cancer-associated fibroblasts; MMP-9, matrix metalloproteinase-9; LVD, lymphatic vessel density; AIS, adenocarcinoma in situ; MIA, minimally invasive adenocarcinoma; IAC, invasive adenocarcinoma.
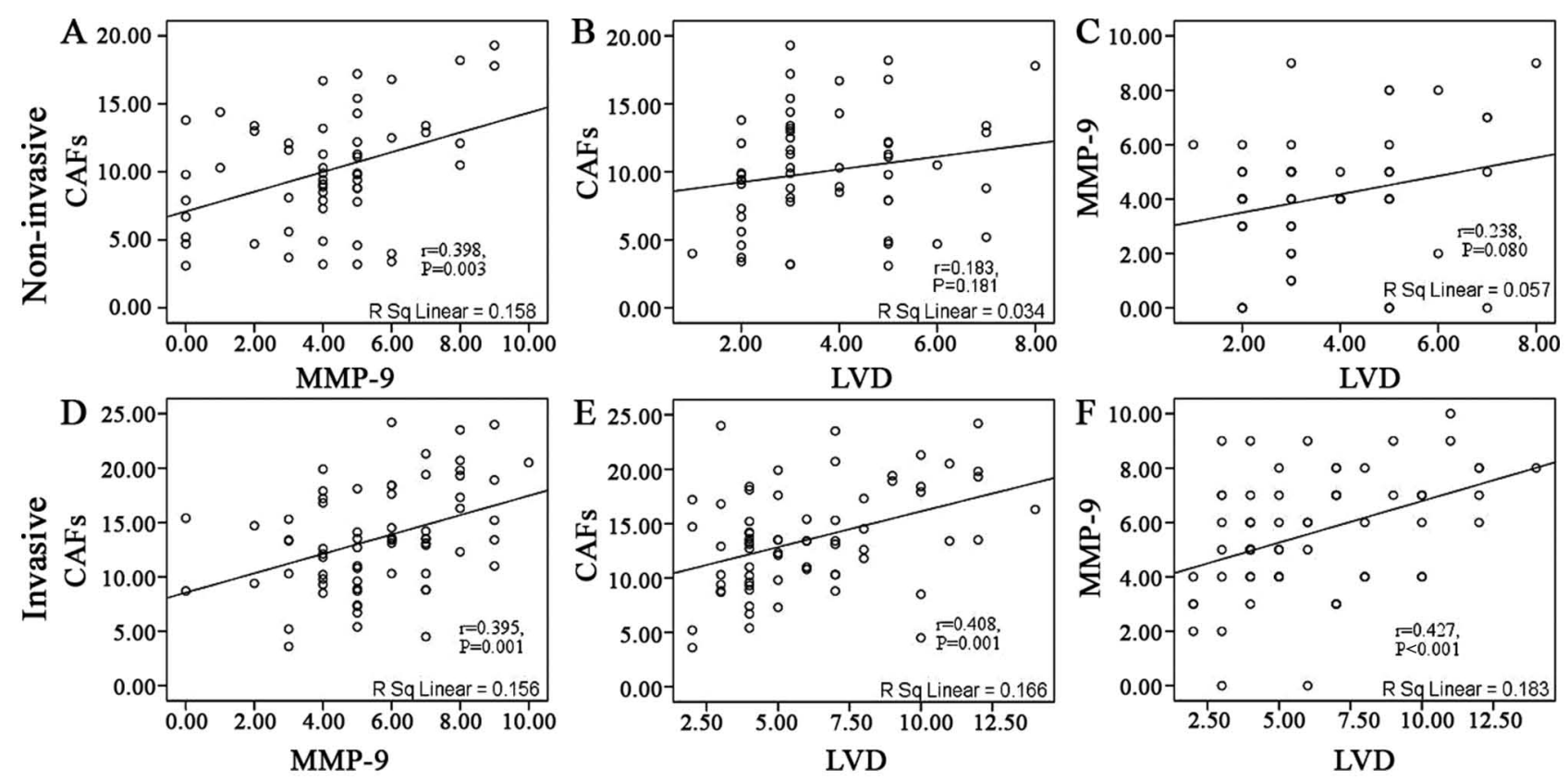

Figure 2. Correlation between CAFs, MMP-9 and LVD in the non-invasive and invasive components. In the non-invasive component, a significant correlation was observed between (A) CAFs and MMP-9, but not between (B) CAFs and LVD, and (C) MMP-9 and LVD. In the invasive component, significant correlations were observed between (D) CAFs and MMP-9, (E) CAFs and LVD, and (F) MMP-9 and LVD. CAFs, cancer-associated fibroblasts; MMP-9, matrix metalloproteinase-9; LVD, lymphatic vessel density.

these findings, the present study revealed that the expression levels of MMP-9 were lower in the non-invasive component compared with in the invasive component. The expression levels of MMP-9 steadily increased in both the non-invasive and invasive components while progressing from AIS to IAC. The current results suggested that the increase in the proportion of CAFs and the expression levels of MMP-9 may be an early event before adenocarcinomas become invasive during tumor progression. The non-invasive component was morphologically similar, but had different invasive potential during progression from AIS to IAC.

Stromal fibroblast-type cells are the most important source of MMPs in mixed bronchioloalveolar carcinoma (31). Using an experimental mouse model, Taguchi et al (32) demonstrated that MMP-9 was expressed in fibroblasts but not in tumor cells. The co-culture of fibroblasts with tumor cells enhanced the expression levels and proteinase activity of MMP-9 (32). In the present study, CAFs in the non-invasive and invasive components were strongly correlated with the expression levels of MMP-9. The aforementioned findings combined with the results of the present study indicated that CAFs may induce MMP-9 expression and promote tumor aggressiveness and progression in the earliest event of lung adenocarcinoma.

Previous studies have revealed that CAFs and MMP-9 influence lymphangiogenesis in ovarian cancer (33), oral squamous cell carcinoma (34) and breast cancer $(35,36)$. Chen et al (37) demonstrated that the abundance of CAFs in lung adenocarcinoma was associated with a higher LVD. This finding was extended in the present study: The LVD increased only in the invasive component while progressing from MIA to IAC, and a positive correlation with CAFs and MMP-9 was detected in the invasive component but not in the non-invasive component. Although further exploration is required for the underlying mechanism, the results of the present study indicated that histopathological patterns should be considered as 
Table V. Differences in CAFs, MMP9 and LVD in the invasive subtypes of patients with invasive adenocarcinoma.

\begin{tabular}{lrrr}
\hline Variable & Acinar + papillary $(\mathrm{n}=55)$ & Solid + micropapillary $(\mathrm{n}=21)$ & P-value \\
\hline CAFs & $12.88 \pm 3.76$ & $16.36 \pm 5.34$ & 0.011 \\
MMP-9 & $5.27 \pm 2.00$ & $6.33 \pm 2.11$ & 0.045 \\
LVD & $5.38 \pm 2.22$ & $8.38 \pm 3.01$ & $<0.001$
\end{tabular}

P-values calculated using a independent-samples Student's t-test. CAFs, cancer-associated fibroblasts; MMP-9, matrix metalloproteinase-9; LVD, lymphatic vessel density.
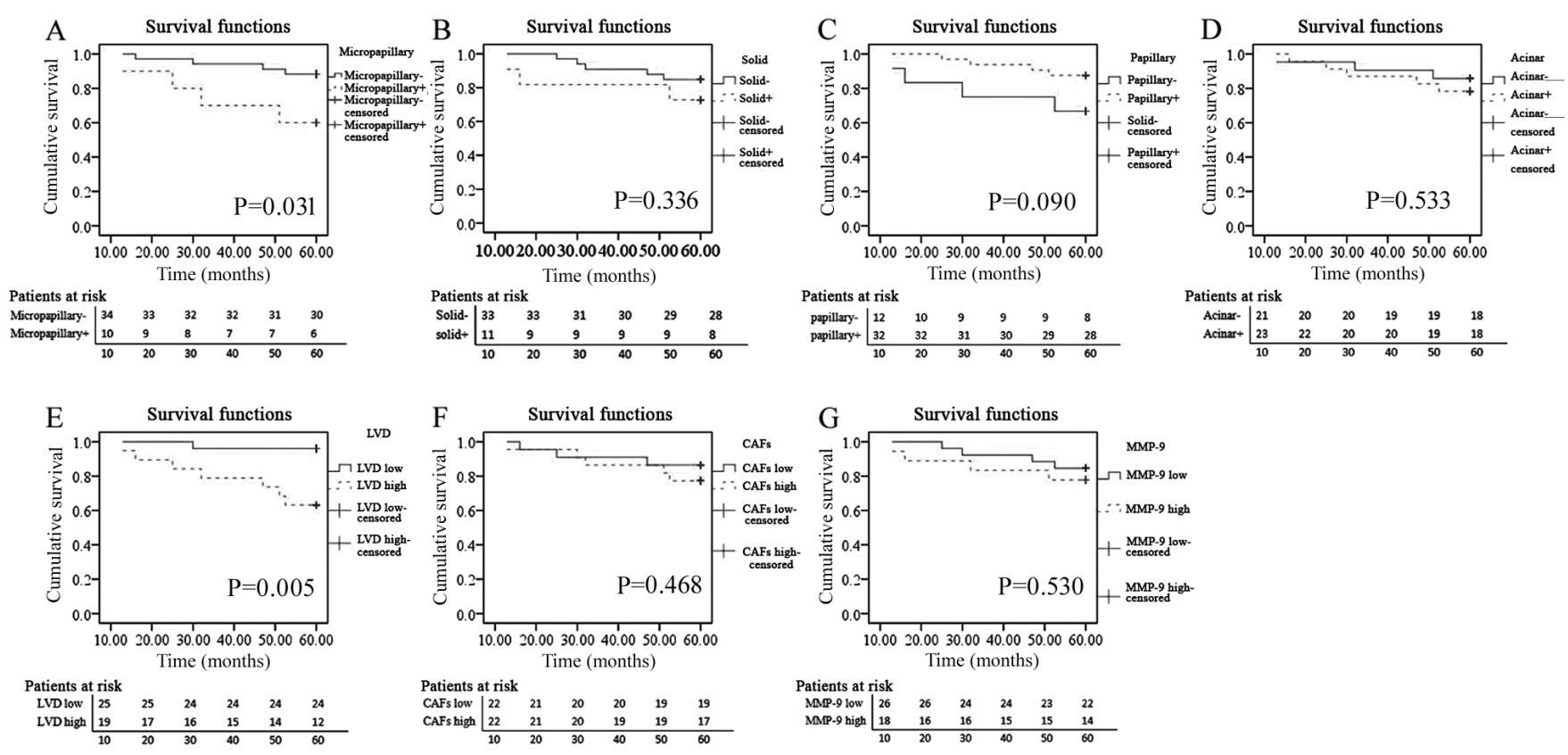

Figure 3. Association between OS and different parameters. Patients were classified into high or low groups based on the median scores of CAFs, MMP-9 and $\operatorname{LVD}(6.0,13.5$ and 5.0, respectively), and positive or negative invasive subtype groups based on whether the invasive subtype is $>5 \%$. Association between OS and (A) micropapillary component ( $\mathrm{P}=0.031)$, (B) solid component $(\mathrm{P}=0.336)$, (C) papillary component ( $\mathrm{P}=0.090)$, (D) acinar component ( $\mathrm{P}=0.533)$, (E) LVD $(\mathrm{P}=0.005),(\mathrm{F}) \mathrm{CAFs}(\mathrm{P}=0.468)$ and $(\mathrm{G})$ MMP-9 $(\mathrm{P}=0.530)$. OS, overall survival; CAFs, cancer-associated fibroblasts; MMP-9, matrix metalloproteinase-9; LVD, lymphatic vessel density.

a prerequisite for exploring the role of CAFs and/or MMP-9 in lymphangiogenesis. Compared with CAFs and MMP-9, the LVD seemed to be a later phenomenon in the progression from AIS to IAC. Once the adenocarcinoma progressed to be invasive, the LVD began to reveal its important role in tumor invasion and metastasis.

Predominant histopathological patterns can reflect the prognosis in patients with lung adenocarcinoma. Patients with solid- or micropapillary-predominant patterns have worse survival rates compared with patients with acinaror papillary- or lepidic-predominant patterns $(38,39)$. Yanagawa et al (40) revealed that patients with solid and/or micropapillary patterns had a poorer prognosis compared with those without solid and/or micropapillary patterns, even if their patterns were not predominant. The results of the present study were consistent with the aforementioned findings, demonstrating that patients with $\geq 5 \%$ micropapillary pattern had a poorer OS rate compared with those with $<5 \%$ micropapillary pattern in stage IA lung adenocarcinoma. However, no significant association was detected between the solid pattern and OS in the present study. Additionally, the current study demonstrated that CAFs, MMP-9 and LVD were all significantly higher in the solid + micropapillary group than in the acinar + papillary group, further supporting the involvement of CAFs, MMP-9 and LVD in tumor invasiveness. However, within stage IA IAC, only the LVD had a significant association with OS. The present result indicated that LVD may be a useful prognostic indicator while selecting therapeutic strategies for patients with early-stage lung adenocarcinoma after surgery. Increased careful follow-up and individual treatments are warranted in cases displaying a high LVD in the invasive component.

The present study had some limitations. First, the sample size was relatively small, and it was a retrospective study conducted at a single institution, which may lead to selection bias. Second, the staining scores of CAFs, MMP-9 and LVD were evaluated using IHC, which provided only a semi-quantitative assessment of protein expression status; additionally, during fixing, processing or staining, the target protein may have been lost (31). Therefore, the use of 
enzyme-linked immunosorbent assay or western blotting with fresh specimens is required in future studies. Third, this was a preliminary study that did not use cell lines or animal models to investigate signaling molecules to further support the results. Additionally, the association between the proportion of subtypes and prognosis was not assessed. Finally, expanding the study to include stage IB-IIA lung adenocarcinoma may have been more conducive to continuous observation of tumor invasive manifestations. These unresolved issues require further study in the future.

In conclusion, the present study revealed that CAFs, MMP-9 and LVD were involved in tumor progression from AIS to IAC. The increase in the proportion of CAFs and the expression levels of MMP-9 may be an early event before adenocarcinomas become invasive. CAFs had a strong correlation with MMP-9 in both non-invasive and invasive components. The LVD increased from MIA to IAC and was strongly associated with CAFs and MMP-9 only in the invasive component. Within stage IA IAC, only the LVD was a prognostic marker for OS. The current findings provided novel insights into the biology of the tumor microenvironment in the early progression of lung adenocarcinoma.

\section{Acknowledgements}

Not applicable.

\section{Funding}

The present study was supported by a grant from the Bureau of Science and Technology of Zhoushan (grant no. 2017B31112).

\section{Availability of data and materials}

The datasets used and/or analyzed in the present study are available from the corresponding author on reasonable request.

\section{Authors' contributions}

CC, BZ and WL designed and performed the study, analyzed the data and wrote the manuscript. ZC, JW, YZ, YW, TD and HL made substantial contributions to the design of the study, acquisition of data, interpretation of data and revision of the manuscript. All authors read and approved the final manuscript.

\section{Ethics approval and consent to participate}

The present study was approved by the Institutional Review Board of Zhoushan Hospital (Zhoushan, China). Informed consent from patients was exempted due to the retrospective nature of the study.

\section{Patient consent for publication}

Not applicable.

\section{Competing interests}

The authors declare that they have no competing interests.

\section{References}

1. Ferlay J, Soerjomataram I, Dikshit R, Eser S, Mathers C, Rebelo M, Parkin DM, Forman D and Bray F: Cancer incidence and mortality worldwide: Sources, methods and major patterns in GLOBOCAN 2012. Int J Cancer 136: E359-E386, 2015.

2. Siegel RL, Miller KD and Jemal A: Cancer statistics. CA Cancer J Clin 69: 7-34, 2019.

3. National Lung Screening Trial Research Team; Aberle DR, Adams AM, Berg CD, Black WC, Clapp JD, Fagerstrom RM, Gareen IF, Gatsonis C, Marcus PM and Sicks JD: Reduced lung-cancer mortality with low-dose computed tomographic screening. N Engl J Med 365: 395-409, 2011.

4. Travis WD, Brambilla E, Noguchi M, Nicholson AG, Geisinger K, Yatabe Y, Powell CA, Beer D, Riely G, Garg K, et al: International association for the study of lung Cancer/American thoracic Society/European respiratory society international multidisciplinary classification of lung adenocarcinoma. Proc Am Thorac Soc 8: 381-385, 2011.

5. Noguchi M: Stepwise progression of pulmonary adenocarcinoma-clinical and molecular implications. Cancer Metastasis Rev 29: 15-21, 2010.

6. Scott WJ, Howington J, Feigenberg S, Movsas B and Pisters K; American College of Chest Physicians: Treatment of non-small cell lung cancer stage I and stage II: ACCP evidence-based clinical practice guidelines (2nd edition). Chest 132 (3 Suppl): 234S-242S, 2007.

7. Joyce JA and Pollard JW: Microenvironmental regulation of metastasis. Nat Rev Cancer 9: 239-252, 2009.

8. Campbell I, Polyak K and Haviv I: Clonal mutations in the cancer-associated fibroblasts: The case against genetic coevolution. Cancer Res 69: 6765-6768, 2009.

9. Ishii G, Ochiai A and Neri S: Phenotypic and functional heterogeneity of cancer-associated fibroblast within the tumor microenvironment. Adv Drug Deliv Rev 99: 186-196, 2016.

10. Feig C, Jones JO, Kraman M, Wells RJ, Deonarine A, Chan DS, Connell CM, Roberts EW, Zhao Q, Caballero OL, et al: Targeting CXCL12 from FAP-expressing carcinoma-associated fibroblasts synergizes with anti-PD-L1 immunotherapy in pancreatic cancer. Proc Natl Acad Sci USA 110: 20212-20217, 2013.

11. Egeblad M, Rasch MG and Weaver VM: Dynamic interplay between the collagen scaffold and tumor evolution. Curr Opin Cell Biol 22: 697-706, 2010.

12. Hanahan D and Weinberg RA: Hallmarks of cancer: The next generation. Cell 144: 646-674, 2011.

13. Ito M, Ishii G, Nagai K, Maeda R, Nakano Y and Ochiai A: Prognostic impact of cancer-associated stromal cells in patients with stage I lung adenocarcinoma. Chest 142: 151-158, 2012.

14. Kawase A, Ishii G, Nagai K, Ito T, Nagano T, Murata Y, Hishida T, Nishimura M, Yoshida J, Suzuki K and Ochiai A: Podoplanin expression by cancer associated fibroblasts predicts poor prognosis of lung adenocarcinoma. Int $\mathbf{J}$ Cancer 123: 1053-1059, 2008.

15. Lu P, Weaver VM and Werb Z: The extracellular matrix: A dynamic niche in cancer progression. J Cell Biol 196: 395-406, 2012.

16. Vandooren J, Van den Steen PE and Opdenakker G: Biochemistry and molecular biology of gelatinase B or matrix metalloproteinase-9 (MMP-9): The next decade. Crit Rev Biochem Mol Biol 48: 222-272, 2013.

17. Ebrahem Q, Chaurasia SS, Vasanji A, Qi JH, Klenotic PA, Cutler A, Asosingh K, Erzurum S and Anand-Apte B: Cross-talk between vascular endothelial growth factor and matrix metalloproteinases in the induction of neovascularization in vivo. Am J Pathol 176: 496-503, 2010.

18. Mira E, Lacalle RA, Buesa JM, de Buitrago GG, Jiménez-Baranda S, Gómez-Moutón C, Martínez-A C and Mañes S: Secreted MMP9 promotes angiogenesis more efficiently than constitutive active MMP9 bound to the tumor cell surface. J Cell Sci 117: 1847-1857, 2004.

19. Cai J, Li R, Xu X, Zhang L, Wu S, Yang T, Fang L, Wu J, Zhu X, $\mathrm{Li} \mathrm{M}$ and Huang Y: URGCP promotes non-small cell lung cancer invasiveness by activating the NF- $\mathrm{KB}-\mathrm{MMP}-9$ pathway. Oncotarget 6: 36489-36504, 2015.

20. Stacker SA, Achen MG, Jussila L, Baldwin ME and Alitalo K: Lymphangiogenesis and cancer metastasis. Nat Rev Cancer 2: 573-583, 2002. 
21. Kadota K, Huang CL, Liu D, Ueno M, Kushida Y, Haba R and Yokomise $\mathrm{H}$ : The clinical significance of lymphangiogenesis and angiogenesis in non-small cell lung cancer patients. Eur J Cancer 44: 1057-1067, 2008.

22. Iwakiri S, Nagai S, Katakura H, Takenaka K, Date H, Wada $H$ and Tanaka F: D2-40-positive lymphatic vessel density is a poor prognostic factor in squamous cell carcinoma of the lung. Ann Surg Oncol 16: 1678-1685, 2009.

23. Travis WD, Brambilla E, Burke AP, Marx A and Nicholson AG, (eds). WHO Classification of Tumours of the Lung, Pleura, Thymus and Heart. 4th edition. Lyon, France, IARC Press, 2015.

24. Hashimoto O, Yoshida M, Koma Y, Yanai T, Hasegawa D, Kosaka Y, Nishimura N and Yokozaki H: Collaboration of cancer-associated fibroblasts and tumour-associated macrophages for neuroblastoma development. J Pathol 240: 211-223, 2016.

25. Ikemura S, Aramaki N, Fujii S, Kirita K, Umemura $S$, Matsumoto S, Yoh K, Niho S, Ohmatsu H, Kuwata T, et al: Changes in the tumor microenvironment during lymphatic metastasis of lung squamous cell carcinoma. Cancer Sci 108: 136-142, 2017.

26. Hu JM, Liu K, Liu JH, Jiang XL, Wang XL, Chen YZ, Li SG, Zou H, Pang LJ, Liu CX, et al: CD163 as a marker of M2 macrophage, contribute to predicte aggressiveness and prognosis of Kazakh esophageal squamous cell carcinoma. Oncotarget 8 : 21526-21538, 2017.

27. Hsu YL, Hung JY, Chiang SY, Jian SF, Wu CY, Lin YS, Tsai YM, Chou SH, Tsai MJ and Kuo PL: Lung cancer-derived galectin-1 contributes to cancer associated fibroblast-mediated cancer progression and immune suppression through TDO2/kynurenine axis. Oncotarget 7: 27584-27598, 2016.

28. Otomo R, Otsubo C, Matsushima-Hibiya Y, Miyazaki M, Tashiro F, Ichikawa H, Kohno T, Ochiya T, Yokota J, Nakagama $\mathrm{H}$, et al: TSPAN12 is a critical factor for cancer-fibroblast cell contact-mediated cancer invasion. Proc Natl Acad Sci USA 111: 18691-18696, 2014.

29. Matsubara D, Morikawa T, Goto A, Nakajima J, Fukayama M and Niki T: Subepithelial myofibroblast in lung adenocarcinoma: A histological indicator of excellent prognosis. Mod Pathol 22: 776-785, 2009.

30. Kodate M, Kasai T, Hashimoto H, Yasumoto K, Iwata $\mathrm{Y}$ and Manabe H: Expression of matrix metalloproteinase (gelatinase) in T1 adenocarcinoma of the lung. Pathol Int 47: 461-469, 1997.

31. Kanomata N, Nakahara R, Oda T, Aoyagi Y, Ishii G, Yokose T, Hasebe T, Nagai K, Yokozaki H and Ochiai A: Expression and localization of mRNAs for matrix metalloproteinases and their inhibitors in mixed bronchioloalveolar carcinomas with invasive components. Mod Pathol 18: 828-837, 2005.
32. Taguchi A, Kawana K, Tomio K, Yamashita A, Isobe Y, Nagasaka K, Koga K, Inoue T, Nishida H, Kojima S, et al: Matrix metalloproteinase (MMP)-9 in cancer-associated fibroblasts (CAFs) is suppressed by omega-3 polyunsaturated fatty acids in vitro and in vivo. PLoS One 9: e89605, 2014.

33. Wei R, Lv M, Li F, Cheng T, Zhang Z, Jiang G, Zhou Y, Gao R, Wei $\mathrm{X}$ and Lou J: Human CAFs promote lymphangiogenesis in ovarian cancer via the Hh-VEGF-C signaling axis. Oncotarget 8: 67315-67328, 2017.

34. Lin NN, Wang P, Zhao D, Zhang FJ, Yang K and Chen R: Significance of oral cancer-associated fibroblasts in angiogenesis, lymphangiogenesis, and tumor invasion in oral squamous cell carcinoma. J Oral Pathol Med 46: 21-30, 2017.

35. Wu QW, Yang QM, Huang YF, She HQ, Liang J, Yang QL and Zhang ZM: Expression and clinical significance of matrix metalloproteinase-9 in lymphatic invasiveness and metastasis of breast cancer. PLoS One 9: e97804, 2014.

36. Cirri P and Chiarugi P: Cancer associated fibroblasts: The dark side of the coin. Am J Cancer Res 1: 482-497, 2011.

37. Chen L, Qin Y, Zhang T, Ding N, Chen Y, Zhang Z and Guo C: Clinical significance of cancer-associated fibroblasts and their correlation with microvessel and lymphatic vessel density in lung adenocarcinoma. J Clin Lab Anal 33: e22832, 2019.

38. Yoshizawa A, Motoi N, Riely GJ, Sima CS, Gerald WL, Kris MG, Park BJ, Rusch VW and Travis WD: Impact of proposed IASLC/ATS/ERS classification of lung adenocarcinoma: Prognostic subgroups and implications for further revision of staging based on analysis of 514 stage I cases. Mod Pathol 24: 653-664, 2011

39. Ujiie H, Kadota K, Chaft JE, Buitrago D, Sima CS, Lee MC, Huang J, Travis WD, Rizk NP, Rudin CM, et al: Solid predominant histologic subtype in resected stage I lung adenocarcinoma is an independent predictor of early, extrathoracic, multisite recurrence and of poor postrecurrence survival. J Clin Oncol 33: 2877-2884, 2015

40. Yanagawa N, Shiono S, Abiko M, Katahira M, Osakabe M and Ogata SY: The Clinical impact of solid and micropapillary patterns in resected lung adenocarcinoma. J Thorac Oncol 11: 1976-1983, 2016

This work is licensed under a Creative Commons Attribution-NonCommercial-NoDerivatives 4.0 International (CC BY-NC-ND 4.0) License. 